EOMmUn: Communication et organisation

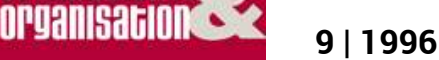

La communication des institutions religieuses

\title{
Les stratégies de communication des protestants français
}

Christian Mesnil

\section{(2) OpenEdition}

1 Journals

Édition électronique

URL : http://journals.openedition.org/communicationorganisation/1841

DOI : 10.4000/communicationorganisation. 1841

ISSN : $1775-3546$

Éditeur

Presses universitaires de Bordeaux

Édition imprimée

Date de publication : 1 mai 1996

ISSN : 1168-5549

Référence électronique

Christian Mesnil, «Les stratégies de communication des protestants français », Communication et organisation [En ligne], 9 | 1996, mis en ligne le 26 mars 2012, consulté le 19 avril 2019. URL : http:// journals.openedition.org/communicationorganisation/1841; DOI : 10.4000/

communicationorganisation. 1841

Ce document a été généré automatiquement le 19 avril 2019

(c) Presses universitaires de Bordeaux 


\title{
Les stratégies de communication des protestants français
}

\author{
Christian Mesnil
}$$
\begin{aligned}
& \text { société où l'information comme la communication passent de plus en plus pa } \\
& \text { - Église discrète qui évite le spectaculaire et les événements médiatiques. }
\end{aligned}
$$

4 - Église largement minoritaire souvent oubliée par le grand public face à la dominante catholique et à la montée de l'Islam.

5 - Église plurielle puisque les protestants français qui sont à peine un million se répartissent en une quinzaine d'églises communiquant chacune à sa manière.

6 - Église de plus en plus disséminée et plus fragilisée que l'ensemble du christianisme européen par la baisse de la pratique religieuse.

7 Face à ces caractéristiques qui pourraient passer pour des handicaps, les protestants français semblent communiquer en interne de façon harmonieuse. Quant à l'image qu'ils transmettent à l'extérieur, un récent sondage (CSA 24 mars - 19 avril 1995) nous révèle un impact grandissant du protestantisme sans rapport avec sa faiblesse en nombre et en prise de parole.

8 La présente réflexion voudrait tenter de comprendre ce phénomène. Pour cette étude qui ne saurait prétendre à l'exhaustivité, une première difficulté réside dans le choix de documents si variés et nombreux qui peuvent nous éclairer. Privilégier les articles parus dans l'hebdomadaire protestant d'actualité Réforme m'a semblé efficace car il croise de façon équilibrée les différentes sensibilités du protestantisme français avec les principaux questionnements ou événements de l'actualité nationale et internationale. Les documents émanant de la Fédération Protestante de France (et en particulier les conclusions de l'audit de communication aimablement confié par son service information communication) ont constitué un second corpus privilégié car cette instance seule a vocation à s'exprimer au nom des différentes églises qui la constituent. 
9 Dans l'évolution des pratiques de communication du protestantisme français peuvent se distinguer quatre étapes qui vont faciliter la structuration de notre réflexion :

10 - Les fondements théologiques et ecclésiaux hérités du passé.

11 - Le contexte des années 70-80 qui voit l'émergence de la communication dans la société française.

12 - Le tournant des années 90 pendant lesquelles s'affirme et se rationalise la communication protestante.

13 - Les tendances et interrogations actuelles : fascination et méfiance.

\section{Les fondements théologiques et ecclésiaux}

Dans son ouvrage L'éthique protestante et l'esprit du capitalisme, le sociologue allemand Max Weber a mis en évidence au début du siècle la contribution historique du protestantisme à l'instauration d'une certaine modernité économique libérale fondée sur le libre échange. Il me semble que cette modernité se situe aussi, et ce dès son origine, dans une capacité à communiquer autrement. En effet, les adeptes de «la religion prétendue réformée » ont proposé implicitement avec plusieurs siècles d'avance une organisation originale basée sur des échanges langagiers plus transparents et proches de la base.

D'abord le courant protestant a été l'un des instigateurs de la lecture de la bible dans le texte sans voir son message altéré par différents intermédiaires. Cet accès d'un plus grand nombre à l'information ne pouvait en toute logique que remettre en cause le mode d'expression hiérarchique de l'église traditionnelle.

16 À l'enseignement ex cathedra d'une doctrine imposée aux fidèles s'est substitué chez les protestants un dialogue plus proche des schémas de communication actuels. Pour qu'il y ait communication, il faut qu'il y ait échange, réciprocité, que le récepteur devienne à son tour émetteur. Figurant parmi les principes les plus novateurs de la Réforme, le sacerdoce universel des croyants instaure une place identique au sein de l'église pour chaque baptisé. Celui-ci ne se contente pas de recevoir des messages mais peut en émettre de plein droit à son tour et l'information doit circuler horizontalement entre laïcs et pasteurs.

17 Enfin, la majorité de l'église réformée française a fonctionné dès ses débuts sous le régime presbytérien synodal. Cette structure pyramidale d'assemblées et d'instances ainsi que ce gouvernement collégial ont permis très tôt une circulation verticale des flux d'information de la base vers le sommet. Il a souvent été affirmé que certains courants importants du protestantisme ont contribué au développement de la démocratie et de la vie associative (loi de 1901). On peut à tout le moins légitimement penser qu'à l'instar de M. Jourdain qui faisait de la prose sans le savoir, les protestants français ont fait figure de précurseurs dans le souci qu'ils avaient d'une communication interne efficace.

Quant à la communication externe, s'il m'est permis de poursuivre l'anachronisme d'une terminologie contemporaine appliquée à une situation bien antérieure, il en va tout autrement. À la modernité et la fluidité des échanges langagiers en interne s'oppose historiquement l'extrême réserve vis-à-vis de l'extérieur.

Point n'est besoin de rappeler d'abord que la communauté protestante fut contrainte à la discrétion dans un contexte plutôt tendu. La révocation de l'Édit de Nantes et les persécutions qui s'en suivirent ont laissé des cicatrices bien au-delà du concordat qui lui 
reconnut au début du XIX siècle le droit d'exister. Un souvenir précis de cette époque reste ancré dans la mémoire collective des protestants français actuels, même lorsqu'ils ne sont pas historiquement descendants d'exilés ou de galériens. Aujourd'hui encore, ils apprécient modérément les coups de projecteur médiatiques lancés sur eux (Heureux comme un protestant en France : L'Express septembre 1982 - Le pouvoir des protestants : Le Matin novembre 1982 - La grande revanche des protestants : Le Point janvier 1996).

La seconde raison historique de cette réserve réside dans les fondements théologiques même de cette église. Le souci calviniste de laisser à Dieu seul la gloire (Soli deo gloria) amène les protestants à refuser instinctivement de se mettre en avant. À cela s'ajoute l'extrême intériorisation du tête à tête avec Dieu rappelé par l'actuel président de l'Église Reformée de France Michel Bertrand: «De nombreux textes bibliques nous disent que Dieu se révèle à nous dans l'abaissement, la faiblesse, la fragilité et non dans les démonstrations de puissance et que souvent la vie devant Dieu prend la forme d'un secret » (Réforme avril 1994).

Enfin une troisième origine à ce déficit de communication externe provient à la fois de cette relation directe avec

Dieu et du régime presbytérien synodal évoqué antérieurement. Le protestantisme français se divise très tôt en trois grands courants : calviniste, luthérien et évangélique. Les dissensions s'accentuent tout au long du $\mathrm{XIX}^{\mathrm{e}}$ siècle et présentent entre autres conséquences le fait que les protestants français ne sont guère consultés au début du siècle par les promoteurs du projet de séparation de l'Église et de l'Etat.

Dans un double souci de représentation pour adopter une position commune face au gouvernement et de rapprochement, les synodes des principales églises se prononcent en faveur de la création d'une fédération qui se constitue dans ses grands principes le 25 octobre 1905 (Plaquette de présentation de la FPF p 4). Finalement la fédération apparaitt dès sa création comme un outil de communication interne et externe. La terminologie qu'elle utilise pour se présenter est assez révélatrice. "Faire se rencontrer et se confronter des traditions et des pratiques différentes (...) prendre en charge un certain nombre de relations (...) représenter le protestantisme (...) intervenir auprès des pouvoirs publics et répondre à leurs sollicitations (...) faire entendre la sensibilité protestante (...) informer régulièrement les médias et publier de nombreux documents sur les sujets d'actualité ».

Une étude comparative des présentations successives que la F.P.F. a produites sur ellemême depuis 1905 mettrait sans doute en avant un lexique et des préoccupations différentes suivant les époques. Qu'y-a-t-il derrière cette terminologie? Un simple toilettage verbal pour être en accord avec le discours ambiant des années 1980 qui a beaucoup mis la communication en avant... et un protestantisme toujours aussi effacé ? Ou au contraire un réel souci de renforcer les liens au sein d'une organisation où l'on agit un peu en ordre dispersé et d'exprimer à l'extérieur des convictions fortes? Si cette dernière hypothèse est la bonne, la F.P.F. a-t-elle mis en place une stratégie pour y parvenir?

\section{Le contexte des années 70 et 80}

Avant de répondre à cette question, il est nécessaire d'une part de faire un choix, d'autre part de rappeler deux évolutions déterminantes dans la société française des années 70. 
26 Le choix consiste à désormais évoquer la communication des protestants français aux sensibilités si variées en privilégiant leur instance fédérative puisque sa vocation première est de parler en leur nom et de les faire communiquer entre eux.

Les deux évolutions vécues en France dans les années 1970 concernent l'une, les pratiques religieuses, l'autre, les pratiques de communication des entreprises et des organisations. La seconde guerre mondiale, avec ses destructions et ses martyrs, avait incité sans doute les Français à accorder une place importante à des questions existentielles, idéologiques et philosophiques (Sartre, Camus...) et la pratique religieuse restait relativement importante. Puis le baby boom d'après guerre amène une nouvelle génération et la fréquentation des églises chute brutalement.

Les protestants français maintiennent relativement bien leurs effectifs, mais s'affaiblissent proportionnellement à la population du pays. De par leur statut déjà minoritaire, la conséquence en est plus dramatique car les réformés français doivent faire face « à un éparpillement beaucoup plus grand sur le territoire national, supprimant des îlots où les protestants représentaient une minorité consistante et pouvaient former une communauté structurée». (Le protestantisme doit-il mourir? de Jean Bauberot, p 27). L'auteur s'appuie sur des statistiques de 1975 mais le titre à la fois provocateur et humoristique de son premier chapitre: «le protestant français; une espèce en voie de disparition » témoigne d'une inquiétude qui reste d'actualité dans les petites paroisses sans renouvellement, ni apport extérieur.

30 La seconde évolution des années 70 concerne la remise en cause de l'autorité (grèves de mai 1968) et la redécouverte du poids de la parole. C'est l'âge d'or des syndicats et des négociations entre organisations syndicales, patronales et institutions politiques. De nombreuses PME se regroupent en structures de production plus importantes pour affronter la concurrence (la société d'assurance le GAN par exemple est constituée en 68). Il devient nécessaire de créer un état d'esprit commun entre les anciens rivaux. Dans certaines entreprises l'autogestion rêvée par la CFDT se concrétise pendant un temps (Société des montres Lip). Les directions du personnel, lorsqu'elles n'étaient pas de simples services de paie, deviennent des services de ressources humaines (cf. - Le Monde 14 décembre 1994, 50 ans du monde de l'entreprise).

31 Les relations avec la presse déjà anciennes se rationalisent. Les entreprises organisent des services de communication, se dotent d'une signalétique, d'un logo, d'une charte graphique. La presse interne se développe dans de nombreuses sociétés.

32 Dialoguer, informer, négocier, transmettre des savoirs deviennent peu à peu des compétences recherchées dans les entreprises. "Le management découvre d'autres ressources au cœur même des rapports humains de travail et l'opinion générale bascule dans une sorte d'attention positive envers la figure d'une entreprise de partenaires où les aliénations et inégalités antérieures céderaient la place à un jeu de négociation plus impliquant, mais plus créateur de développement ».

33 Ce fragment de la conclusion de Renaud Sainsaulieu (Sociologie de l'organisation de l'entreprise $p$ 377) résume bien ce qu'il qualifie d'évolution impressionnante des représentations collectives. Cette évolution va concerner également la communication et va passer des entreprises aux organisations. Les ministères, les collectivités territoriales ou locales, les partis politiques, les grandes associations humanitaires créent à leur tour dans les années 1980 des services de communication et/ou s'adressent à des agences spécialisées. 

de France. Elle organise en mars 1988 un colloque à Paris dont l'intitulé se passe de commentaire: "Communication chrétienne à quel prix? Marketing du message évangélique? Questionnement éthique ». Un conseil en communication : Gil Kressmann (Directeur associé de l'agence Soprano à l'époque) propose dans l'hebdomadaire Réforme du 5 mars 1988 une réflexion au titre aussi évocateur : «Ne sont-elles pas aussi des entreprises? Pour un marketing des églises ». Son discours est typique du transfert de la terminologie et des préoccupations du monde de l'entreprise à celui des organisations. Comme pour n'importe quel produit, il est question de cycle de vie avec lancement (période prophétique), phase de croissance (ère des bâtisseurs), maturité (bureaucratisation, enjeux de pouvoir) et déclin possible... La concurrence directe et indirecte est évoquée à propos de la communication. "Le chrétien est assailli de messages de la part de son entreprise, de la part des médias, des hommes politiques... Il reçoit près de six cent messages publicitaires par jour. Le message diffusé par la paroisse, l'église est donc en concurrence avec les autres qui ont l'avantage d'être souvent plus simples quant au fond. Raison de plus pour être encore plus performant que la « concurrence » en abordant la communication de l'église en véritables professionnels. Car les églises protestantes semblent avancer à reculons dans cette société d'information. L'église calviniste est la dernière de la classe. Si nous ne nous réveillons pas rapidement en y consacrant les moyens nécessaires, nous serons balayés ».

Crainte de disparaître devant le déclin de la pratique religieuse et, à l'instar des entreprises, nécessité de communiquer pour continuer à exister semblent constituer deux leitmotiv chez les protestants français à cette époque.

Le jugement de Gil Kressmann est bien sévère car la dernière de la classe a créé un poste consacré à l'information communication dès les années 1960 au sein de la F.P.F. et le bulletin d'information protestant est mis en place à la même époque. La volonté de ne pas marcher à reculons existe mais les moyens dont dispose la fédération sont limités aux cotisations de ses adhérents et à des dons sans rapport avec les tarifs des agences de conseil en communication... Sans rapport ? Une entreprise anonyme va aider à plusieurs reprises la fédération à condition que sa générosité soit affectée à la communication des services de la rue de Clichy. Cette belle histoire survenue voici une dizaine d'années illustre à la fois ce transfert des mentalités de l'entreprise vers les organisations y compris religieuses, et cette évolution des préoccupations car un tel don aurait probablement été fléché très différemment trente ans auparavant, (siège de F.P.F. à Paris).

\section{Le tournant des années 90}

Les interrogations et les réflexions des années antérieures se concrétisent. La fédération protestante de France élabore un dossier de présentation comportant dix huit feuillets distincts. Leur contenu informatif autonome mais précis, précédé de titres présentant le thème et l'idée Principale utilise les méthodes de professionnels. La présence en apparence banale d'un curriculum vitae de l'actuel président Jacques Stewart (feuillet $\mathrm{n}$ -5) constitue une singulière évolution dans une communauté dont l'un des fondements évoqué antérieurement est de mettre Dieu seul en avant.

Cette plaquette est complétée entre autres documents par un minifascicule de seize pages destiné au grand public. Il explique de façon attractive l'historique, les rouages, les

Communication et organisation, 9 | 2012 
adhérents, la charte de la fédération et remplace avantageusement des documents plus ternes.

Dès 1991, la F.P.F se dote d'un logo assorti d'un guide d'utilisation pour en expliquer à ses membres l'intérêt autant que le mode d'emploi. Il y est question d'une harmonisation de la communication interne et externe, mais aussi des messages visuels véhiculés par les médias, de la nécessité de visibilité, de mémorisation par des signes forts, simples et répétés. Quelle évolution pour des croyants qui se sont toujours méfiés des images...

Enfin au cours de cette même année un cabinet de consultants est sollicité pour un audit. Ses conclusions méritent une analyse approfondie.

41 Tout d'abord, cet audit dresse un constat classique de la pluralité des protestantismes français - des sensibilités très diverses qui rendent délicate la communication interne et problématique la visibilité externe face au mastodonte catholique. Il y a les majoritaires (Église réformée de France) et les autres, ceux qui font partie de la fédération et ceux qui voudraient y entrer avec tout ce que cela suppose de confrontation, de renouvellement, de dialogue interne mais aussi de risque d'incompréhension, voire même de conflit. « La cohésion interne est fragile ( $\mathrm{p} 4)$. L'union est vulnérable ( $\mathrm{p} 9$ ). L'élément fédérateur doit dépasser le niveau du label officiel et concerner un grand projet mobilisateur ( $p$ 16). Lancez une grande consultation en interne (p 17) ».

42 Conséquence logique de ce constat, la même exhortation se décline tout au long du document: «Réveillez-vous! Pour bien communiquer, il faudrait d'abord le vouloir. $\mathrm{Ne}$ vous plaignez pas d'être souvent ignorés par les médias et parfois traités comme quantité négligeable par les catholiques » ( $\mathrm{p}$ 5). Le point d'orgue en est la cinquième et dernière prise de position de l'audit qui occupe plus du quart de celui-ci et concerne la communication de la fédération. Un diagnostic rapide (une page sur huit) est dressé sur un grand problème : Votre communication interne souffre d'athérosclérose aiguë. Chacun est un peu dans son coin réfléchissant bien mais communiquant mal (p 22). Puis est envisagée sur six pages la communication externe d'une façon tout à fait caractéristique.

En effet quatre pages sur six n'évoquent la communication des églises protestantes que par rapport à celle de l'église catholique romaine ou française. Les sous-titre sont clairs :

$44-55$ : Soyez différents des catholiques dans votre communication (...) $\mathrm{Ne}$ communiquez pas comme les catholiques ( $\mathrm{p} 24$ ).

$45-56$ : La communication catholique est d'abord magistérielle. Celle de la fédération doit privilégier le dialogue ( $\mathrm{p} 25)$.

$46-57$ : La communication catholique est d'abord diocésaine. Celle de la fédération doit être nationale (p 26).

47 - 58: La communication catholique française repose avant tout sur une voix, celle du Pape. Celle de la fédération doit être à la fois personnelle et plurielle (p 27). En fait l'aboutissement de cet audit n'a rien d'étonnant quand on en relit le début qui présente en quelque sorte sa problématique. "Tel le badaud parisien des Lettres Persanes se demandant comment peut-on être persan? tout papiste, placé en situation d'audit de la F.P.F ne peut que s'exclamer: comment peut-on être protestant?» Suivent dès la première page plusieurs allusions significatives: «catholicisme majoritaire " " conférences des évêques » - " hiérarchie catholique, ses diocèses, ses paroisses ".

48 Certes le cabinet Garnier Parisot doit bien connaître les catholiques pour avoir travaillé avec plusieurs de ses organisations (Secours catholique, CCFD). Par ailleurs, il existe en 
expression une base fondamentale selon laquelle plus les éléments sont contrastés, mieux ils se mettent en valeur mutuellement. Peut-être que cette mise en parallèle Presque systématique avec les pratiques les plus visibles de la communauté religieuse dominante procède simplement d'une préoccupation didactique. Il existe enfin une troisième explication possible, le souci de préserver ou reconquérir une spécificité que l'œcuménisme tend à gommer. "En donnant dans l'œcuménisme, vous vous êtes mis à l'unisson, vous vous êtes dilués au point que vous donnez parfois l'impression de douter de votre spécificité » (p 3).

Pourtant le trait est parfois forcé à un point tel qu'on se croirait revenu aux plus beaux jours de la lutte acharnée du XIX ${ }^{e}$ siècle entre les deux propagandes d'églises. Après avoir pris la précaution de préciser «je veux dire Par là, non pas que les catholiques communiquent mal - c'est un autre problème - ou qu'il faut donner dans l'anticatholicisme - ce serait stupide» (p 24) on peut lire entre autres une douzaine de lignes plus bas ( $\mathrm{p}$ 25). «La communication catholique est abondante, lente et lourde, souvent à huis clos. Plus que de la communication, c'est de l'enseignement diffusé du haut vers le bas, paternel, un tantinet infantilisant, avec, périodiquement un vieux réflexe pavlovien de père fouettard toujours prêt à foncer sur le chiffon rouge des provocations du sexe (exemple : publicité Benetton) ».

Définir sa communication non pas par rapport à soi-même mais par rapport à celle d'une autre organisation religieuse procède certes d'un certain sens du positionnement face à la concurrence. Mais pourquoi l'audit n'évoque-t-il pas la communication d'autres communautés religieuses reconnues en France (israélites et musulmans) par rapport auxquelles le protestantisme pourrait d'autant mieux se situer que le statut de communautés minoritaires est comparable? Par ailleurs, pourquoi ne pas réfléchir par rapport aux protestants français eux-mêmes ? La situation de leur presse est à cet égard tout à fait caractéristique. L'édition 1996 de l'annuaire de la France protestante ne répertorie pas moins de 123 titres. Le million de protestants est à l'origine de 46 revues et bulletins, 6 hebdomadaires et périodiques, 16 journaux de région et d'unions d'églises, 24 revues d'évangélisation et de mission, 12 publications pour la jeunesse, 8 publications pour le personnel médical, les malades et les handicapés, 11 journaux consistoriaux et locaux. Le début de l'audit exhortait la fédération en ces termes : «Faites preuve d'une réelle volonté de communiquer en tenant compte des règles du jeu médiatique aujourd'hui. Vos ancêtres n'ont pas boudé l'imprimerie, ne vous privez pas des médias » (p 5).

51 D'une certaine façon, cette réelle volonté de communiquer, les protestants français ne l'ont que trop. Au lieu de se priver des médias, ils en usent et en abusent au point d'aboutir à un éparpillement préjudiciable à cette communication. Ce type de situation qui concerne le protestantisme français lui-même aurait pu, me semble-t-il, être posé dans cet audit avec des préconisations qui permettent non de tarir un tel foisonnement, mais de le restructurer, de lui fournir une aide logistique, technique pour en augmenter l'efficacité.

Les audités ont-ils consciemment ou non convaincu leurs interviewers du bien fondé de cette perspective ancienne ? Les consultants l'ont-ils reprise sans le savoir? Avaient-ils le souci (fréquent...) de positiver leur client ou observaient-ils la vieille méthode qui consiste d'une part à conforter l'organisation dans certaines attitudes pour mieux la faire évoluer sur d'autres plans? Le cadre limité de cette étude ne permet pas d'étendre la réflexion aux pratiques des consultants et à l'analyse des audits produits. Mais le fait de 
retrouver chez des spécialistes en principe neutres et extérieurs de vieux réflexes bien connus des commanditaires de l'audit est pour le moins singulier.

Par ailleurs les consultants ont assorti leurs conseils méthodologiques de propositions d'action précises. Pour classiques qu'elles puissent paraitre aux yeux d'un dircom actuel (affichettes, gadgets, bandes dessinées, questionnaires à choix multiples) elles n'en présentent pas moins une évolution importante eu égard au contexte dans lequel ces suggestions ont été formulées.

Aujourd'hui, la communication de la F.P.F. est davantage personnalisée. La photo de l'actuel président figure sur de nombreux supports. Cela aurait été impensable il y a vingt ans à l'époque où mettre quelqu'un en avant était encore considéré comme une dérive choquante. L'audit préconisait « une structure de guérilla, capable de se transformer momentanément en cellule de crise, légère, (...) rapide (réactions aux coups d'épingles) ». Des communiqués de presse A4 recto presque à chaud sont publiés par la fédération, comme celui paru le 27 juin 1993 à propos du débat sur le code de la nationalité et l'immigration ou celui paru le 12 février 1996 à la suite du rapport parlementaire sur les sectes en France.

55 Cet audit constitue une étape significative dans le cheminement du protestantisme français par rapport aux pratiques communicationnelles de la fin de ce siècle. On peut y distinguer au moins quatre caractéristiques :

56 - L'humilité d'une organisation qui a conscience de méconnaître le monde de la communication, ses contraintes et sa logique.

57 - Le pragmatisme qui la pousse à s'adapter et à faire appel à des consultants pour l'aider dans son entreprise.

58 - Le souci de tirer profit de l'audit et de suivre de son mieux les principales recommandations, en dépit de la modestie de ses moyens.

59 - Enfin un certain empirisme reste perceptible comme si des résistances entravaient cette marche en avant.

\section{Les tendances et interrogations actuelles}

60 Corriger ce qu'elle considère comme un déficit en communication, faire entendre la parole protestante dans les médias est devenu un souci prioritaire du protestantisme français. Le président de la F.P.F Jacques Stewart le soulignait dans une interview au journal Réforme après sa réélection (4 février 1995) «J'espère que la politique de communication développée depuis une dizaine d'années à la fédération va continuer de porter ses fruits et que nous serons de plus en plus sollicités. Mais il faut prendre beaucoup d'initiatives afin que chacun sache que les protestants existent. Je suis persuadé que beaucoup de gens ignorent même l'existence du protestantisme dans notre société. Celui-ci est tellement muet... tellement timide».

61 Les initiatives se multiplient en effet pour accéder aux circuits médiatiques. Sur quatrevingt treize radios locales œcuméniques répertoriées par la fédération, trente-deux sont d'obédience protestante. Des serveurs Minitel se sont mis en place.

À chaque fois qu'ils le peuvent les représentants du protestantisme participent aux émissions télévisées ou radiophoniques. 
63 Mais cette démarche suppose en amont de disposer d'un message clair, si possible en cohérence avec les multiples sensibilités présentes au sein de la F.P.F.

À l'inverse d'une fédération syndicale qui représente des courants de pensée convergents, la fédération protestante regroupe des églises sans unanimité, avec des traditions différentes. L'étude des « règles d'élaboration des positions éthiques protestantes» (juin 1988) qui figurent au début du livre blanc paru en 1995 est assez significative.

Point 5: «La forme spécifique des positions protestantes serait en deux volets: construction d'un possible compromis ou accord (toujours plus profond et plus large qu'on ne l'imagine souvent), expression d'un éventuel différend (la pluralité est un témoignage si elle est cohérente et si le différend fait apparaître des différences et des questions nouvelles) ». Dans la réalité, même si les positions communes existent, il n'est pas toujours simple de communiquer sur les sujets qui ne font pas l'unanimité. Le protestantisme français, pas plus que les autres organisations religieuses ne doit être exempt de querelles. Elles sont feutrées, sans excommunication ${ }^{\circ} \mathfrak{u}$ démission forcée (affaire Gaillot) mais elles existent et amènent sans doute certains protestants à quitter leur église... Olivier Abel, président de la commission d'éthique de la fédération, précise dans le préambule du livre blanc. «Un texte adopté par le conseil n'est pas forcément plus unanime qu'un autre et il arrive qu'il soit adopté malgré une forte réticence ». Il doit en être de même pour la fédération dont les porte-parole (le Président, le service d'information communication) doivent observer une certaine prudence. Sur la bioethique, l'interruption volontaire de grossesse, l'homosexualité, les positions évangélisques et pentecôtistes diffèrent. Deux solutions existent alors : ne pas parler des choses qui fâchent ou évoquer les différents Points de vue.

La seconde solution a des conséquences inattendues. La participation par exemple de la fédération au comité de préparation de l'année Clovis aux côtés d'hommes politiques, historiens, évêques... est loin de faire actuellement l'unanimité chez les protestants si l'on en croit la rubrique lecteurs de Réforme. Or ce désaccord n'apparait guère dans les médias français parce qu'il paraît dérisoire ou que les querelles des minoritaires n'intéressent pas.

67 Par ailleurs, certaines différences n'apparaissent pas comme polémiques aux yeux des médias. Sur de nombreuses questions cruciales pour la société de notre temps (écologie, euthanasie, sida, accueil de l'étranger...) les positions sont mesurées. Les protestants français observent non sans raison que l'opinion publique accueille plus favorablement des positions ouvertes, non définitives. «Souvent il s'agit ainsi moins de répondre à une question que de substituer à un débat qui nous semble mauvais ou faux, un débat meilleur ou plus juste. Notre communauté se définit parfois mieux par notre manière de débattre que par notre avis final» (Règle d'élaboration des positions ethiques protestantes $n^{\circ} 5$ ). Là encore singulière évolution de mentalité qui relativise l'importance du fond et du rationnel au profit de la forme, du relationnel et de la façon de communiquer.

Enfin si le protestantisme français (qui contrairement aux apparences, n'est pas silencieux) ne fait pas sensation, c'est aussi parce que ses positions sont trop proches de l'opinion publique. Partisan de la laïcité, structuré de façon démocratique, ayant toujours eu des pasteurs mariés, ordonnant les femmes depuis la seconde guerre mondiale, acceptant le divorce, la contraception, n'ayant aucun problème moral ou théologique avec le préservatif, comment peut-il se démarquer, faire parler de lui ? Alain Duhamel écrit au sujet de la spécificité protestante que «le protestantisme français correspond 
tant aux aspirations, aux inquiétudes et aux équivoques de la société en cette fin du $\mathrm{XX}^{\mathrm{e}}$ siècle, à ses contradictions aussi qu'il peut aussi bien s'y forger que s'y perdre » Le Point 27 janvier 96. Curieux paradoxe que celui de la communication d'une minorité qui aurait été tellement performante (le « levain « huguenot...) qu'elle serait vouée à ne plus être entendue.

69 À en croire la liste des thèmes que la commission d'éthique compte traiter prochainement: drogue, médias et journalisme, chômage, justice, voiture, logement, écologie, don et greffe d'organe (livre blanc p 38) les protestants français sont décidés à continuer sur leur lancée et à poursuivre la diffusion d'une parole réformée auprès d'une opinion française qui ne la remarque guère. Pourquoi ?

70 Il y a d'abord dans cette ténacité le sens profond d'une mission: "agir, intervenir pour changer le monde » et la volonté d'exprimer une foi, de porter un regard sur l'existence dans une société de plus en plus matérialiste: «nous sommes placés ensemble dans la responsabilité ouverte par le texte biblique et par l'évangile (Règles d'élaboration des positions éthiques protestantes)».

71 Mais tout se passe comme si une retombée non négligeable de cette prise de parole dans les médias sur les sujets de société se situait au sein du protestantisme français lui-même. En essayant de percer (difficilement) à l'externe, un «bénéfice «substantiel est de toute façon retiré dans la communication interne de l'organisation. Les fonctionnements ecclésiaux étaient probablement autrefois davantage préoccupés par les traditions internes, le règlement des institutions, les rapports diplomatiques entre les uns et les autres.

Grâce à ses prises de position de type communiqué de presse ou livre blanc, les convictions théologiques, ecclésiologiques, ethiques se clarifient peu à peu entre les partenaires de la F.P.F et le souci d'une communication interne plus performante progresse, «il n'y a pas de double langage possible, c'est en parlant à l'extérieur des églises que l'on est entendu en leur sein». Cette partie du point sept des règles d'élaboration des positions éthiques Protestantes participe un peu des deux éléments de réponse proposés ci-dessus.

Le dernier point de cette étude concerne les relations complexes que le protestantisme français entretient avec le monde des médias. Nous avons étudié antérieurement son souci constant d'être partie prenante d'un système. Or pour y intervenir de façon efficace, il faut utiliser des moyens que la « culture » protestante a du mal à intégrer.

Les protestants français n'ont pas de figure de proue médiatique (Pape ou évêques, fusent-ils dissidents), de personnage comme l'Abbé Pierre ou Sœur Emmanuelle. Ils disposent certes de quelques lieux de mémoire (les musées protestants), de quelques rassemblements ( $\mathrm{du}$ culte annuel du musée du Désert au Mas Soubeyran dans les Cévennes réunissant 20000 personnes à d'autres rassemblements plus modestes comme celui de la Pierre plantée dans le Tarn). Mais cela est sans commune mesure avec le folklore, les symboles, les pèlerinages et rassemblements, les lieux sacrés et patrimoines artistiques d'autres communautés religieuses.

La campagne d'évangélisation du pasteur Billy Graham en 1986 au palais omnisports de Bercy retransmise par satellite dans plusieurs villes françaises et à Genève avait à l'époque suscité beaucoup de controverses chez les protestants français (cf. Quand un américain se permet d'évangéliser la France: chapitre 9 dans Le protestantisme doit-il mourir?) La suspicion transparaît toujours dès qu'une vedette est mise en avant. 
L'exposition répétée à certains types d'images fait que la communication est réussie, mais l'efficacité est-elle le seul critère? La manipulation télévisuelle, son message réducteur, l'uniformisation de la pensée, la passivité du téléspectateur sont des conséquences maintes fois dénoncées et pas seulement par les protestants. Mais certains d'entre eux comme Jacques Ellul ont été parmi les premiers à souligner les risques encourus à cause de l'audiovisuel (La parole humiliée).

L'actuel président de l'Église reformée de France Michel Bertrand avait, me semble-t-il, résumé de façon très claire l'évolution en cours dans une interview parue dans Réforme en avril 1994. « Il est difficile d'être une église de la parole dans une civilisation de l'image et Régis Debray a bien montré comment le protestantisme pouvait souffrir aujourd'hui de la contre réforme cathodique »(...). Ce n'est pas parce que c'est difficile qu'il faut trop vite renoncer. L'image est un langage que nous pouvons apprendre à lire et à utiliser de manière critique. Il faut se réjouir des efforts faits dans ce sens au sein des églises protestantes (...).

77 Cette évolution est confirmée par de nombreuses pratiques de la fédération évoquées antérieurement (logo, personnalisation) et s'appuie sur une réflexion de ses intellectuels (notamment les travaux de Jérôme Cottin: thèse de doctorat en théologie soutenue en 1993, intitulée Parole de Dieu et signes visuels, évaluation et redécouverte de l'image en théologie protestante dans le contexte d'une société médiatique, ainsi que Le regard et la parole, une théologie protestante de l'image Labor et Fides 1994).

78 Le discours sur les images qui peuvent signifier plus qu'elles ne montrent, qu'il faut apprendre à décrypter avec un certain esprit critique n'est pas vraiment neuf pour ceux qui s'intéressent aux sciences de l'information et de la communication. Pourtant il ne va pas de soi dans une communauté où le livre était traditionnellement perçu comme le seul objet culturel permettant la liberté, la réflexion personnelle et l'approfondissement. L'image immédiate, publicitaire, envahissant les espaces publics et privés reste probablement indigne aux yeux d'une certaine fraction de la communauté protestante. La vigueur des réserves énoncées par le Président de l'E.R.F. dans l'interview citée plus haut à propos de l'utilisation de l'image est à cet égard significative. « Notre quête de visibilité médiatique ne saurait se faire au détriment de nos choix théologiques et ecclésiologiques. La fidélité à l'église ne se mesure pas à l'audimat (...). Être présent dans les médias ne signifie pas obéir à leur logique. Nous n'avons pas à vendre notre âme pour répondre à leurs exigences ».

79 La communauté protestante française est vraisemblablement riche de nuances avec des fondamentalistes, des traditionalistes, des élitistes fiers de leur appartenance sociale et culturelle où le livre jouait un rôle prépondérant.

80 Ce sujet doit être particulièrement délicat à aborder pour les protestants car derrière les images se profilent les idoles et on sait combien cette église est sensible sur ce point qui constitue un des fondements de sa théologie. Les responsables du protestantisme français n'ont guère d'autre choix que celui d'un discours dialectique complexe quand ils communiquent sur cette question afin de réussir une évolution tout en maintenant l'union, ce qui n'est jamais simple.

81 Actuellement la réflexion des protestants français semble évoluer de façon plus globale vers les médias en général et le débat est peut-être moins polémique que sur le rôle de l'image. Une conférence débat s'est déroulée en octobre 1995 à Montpellier avec un commentateur de presse célèbre, Yvan Levai, de France Inter. Elle avait pour thème les 
protestants face au défi des médias et a alterné les témoignages d'une foi en la parole et "les signes d'une évolution qui risque de nous amener au crépuscule de l'écrit par l'effet dévastateur de la télévision et des techniques modernes de communication, internet me rapproche de mon lointain et m'éloigne de mon prochain ". (Compte rendu de J. Agulhon dans Réforme du 21 octobre 1995). Michel Serres était interrogé quelques mois plus tôt suite à son livre Atlas sur un thème proche : du bon usage des médias. «Presque toujours les informations désormais ne sont que de la publicité, c'est ce que j'ai appelé le métamensonge ». Il évoque l'accès à des myriades d'informations, le tri nécessaire, la transformation du lien social pour conclure sur une position médiane : «Il existe toujours des gens pour faire le travail du deuil de la culture morte sans voir la naissance d'une nouvelle culture et il existe des enthousiastes de la culture nouvelle qui ne comprennent pas ce qu'elle détruit. Il faut tenir des deux attitudes ». (Propos recueillis par M.L. Bernasconi - Réforme du 4 février 1995) mais quel que soit le spécialiste interpellé la réponse est pour le moment toujours ambiguë, riche d'inquiétude et de promesse. Les nouveaux modes de communication (Internet, Numéris, Langages virtuels) annoncent des bouleversements. Les disparités seront de plus en plus grandes entre ceux qui maitrisent les nouveaux processus et les autres. La solitude, la manipulation menacent de transformer les acteurs en agents. Les protestants français semblent particulièrement préoccupés par cette évolution. Leurs médias, Réforme en particulier, rendent compte régulièrement de l'évolution de la situation, interrogent professionnels et chercheurs.

\section{Pour conclure}

La modernité communicationnelle des protestants français était à l'origine davantage présente à l'interne qu'à l'externe. La situation s'est progressivement inversée à cause de ses composantes variées et de sa position de plus en plus minoritaire. Pour ne pas démissionner et risquer de se dissoudre, ils devaient impérativement faire entendre leur voix d'une autre manière.

Parallèlement à l'évolution de la société économique, politique, civile vers plus de communication, ils se sont donc adaptés. La démarche utilisée s'est voulue à la fois rigoureuse, appliquée et... un peu empirique, mais elle porte du fruit. La fédération protestante de France semble maintenant en éveil constant, prête à communiquer au coup par coup dès que le besoin ou l'occasion se présente malgré l'absence de stratégie ou d'échéancier prédéfinis. Cela ne lui réussit finalement pas si mal car son message, tout en n'ayant jamais un retentissement médiatique considérable, passe bien dans l'opinion publique française qui lui accorde bienveillance et estime.

84 L'effort a été plus important qu'un regard extérieur Pourrait le penser, à cause de la modestie de ses moyens, de ses divisions et de certaines valeurs théologiques ou ecclésiales apparemment peu compatibles avec les pratiques des médias.

L'effort est à poursuivre d'autant plus que l'on rentre dans un univers médiatique très ambigu, omniprésent et dont l'évolution vertigineuse laisse entrevoir et des champs de possibilités prometteurs et des risques de manipulation encore plus inquiétants.

Loin de se laisser décourager par l'ampleur et la rapidité avec laquelle les nouvelles technologies de l'information et de la communication envahissent notre société, la "petite "fédération des protestants de France fait ainsi preuve d'un singulier dynamisme. 
ui qui consiste (tout en confortant des pratiques communicationnelles adaptées à la société moderne) à s'interroger de plus en plus au nom des capacités de choix et de réflexion humaine qui lui tiennent tant à cœur sur le sens et les formidables enjeux de la communication du futur.

\section{RÉSUMÉS}

En dépit de caractéristiques qui pourraient passer pour des handicaps (minoritaires, divisés, réservés, disséminés...) les protestants français semblent communiquer de façon efficace. En réalité leurs fondements théologiques et ecclésiaux comportent également des atouts. Par ailleurs l'importance grandissante de la communication dans les entreprises et organisations les ont amenés à modifier profondément leurs modes d'intervention auprès des médias. De fait le protestantisme français communique maintenant avec des outils et des comportements professionnels autant qu'au coup par coup en amateur. Cette double démarche peut s'expliquer à la fois par la modestie des moyens matériels disponibles et par les réticences de certains de ses membres vis-à-vis d'attitudes qui bouleversent leur culture. Ce point de vue est conforté par la formidable progression des nouvelles technologies de l'information et de la communication. Elles ont pour conséquence une amplification de la réflexion des protestants français sur les défis et le bon usage des nouveaux médias.

In spite of characteristics which could be construed as hindrances (they are a minority, they are divided, reserved, spread out...) French Protestants seem to able communicate in an efficient way. Actually, their theological and ecclesial bases also their assets. Furthermore, the growing importance of communication within companies and organisations has led them to modify in depth the ways in which they interact with the media. In fact, French Protestantism now communicate with professional tools and behaviours as well as, should the need arise, in an amteurish way. This double process can be explained both by the scantiness of the material means available to them and by the reservations some of theirs members have about attitudes which cause upheaval within their culture. These reservations are not subsiding with the extraordinary progression of the new information and communication technologies. As a result, the French Protestants reflexion on the challenges and the good uses of the new media is intensifying.

\section{AUTEUR}

\section{CHRISTIAN MESNIL}

Maître de conférence en sciences de l'information et de la communication à l'Université du Littoral - Enseignant au département Gestion des Entreprises et des Administrations (IUT. de Calais) et à l'ENIC : Ecole nouvelle d'ingénieurs en communication (Villeneuve d'Ascq). 